\title{
Seleção de genótipos de capim-elefante (Pennisetum purpureum) utilizando a metodologia REML/BLUP
}

\section{Selection of elephant grass genotypes (Pennisetum purpureum) by methodology REML/BLUP}

\author{
Bruna R. da S. Menezes ${ }^{1^{\star}}$, Rogério F. Daher ${ }^{2}$, Geraldo de A. Gravina², Verônica B. Silva², Erina \\ V. Rodrigues², Maria L. F. Oliveira², Flávio D. Tardin³, Niraldo J. Ponciano², Maria do S. B. de \\ Araújo² e Avelino dos S. Rocha²
}

\author{
1 Universidade Federal Rural do Rio de Janeiro, Departamento de Genética, CEP 23890-000, Seropédica, Rio de Janeiro, Brasil; \\ ZUniversidade Estadual do Norte Fluminense Darcy Ribeiro, Laboratório de Engenharia Agrícola, CEP 28013-602, Campos dos Goytacazes, Rio de Janeiro, Brasil; \\ ${ }^{3}$ Empresa Brasileira de Pesquisa Agropecuária, CEP 78550-970, Sinop, Mato Grosso, Brasil. \\ ( ${ }^{\star} E$-mail:brunarafamenezes@hotmail.com) \\ http://dx.doi.org/10.19084/RCA15073
}

Recebido/received: 2015.06.21

Aceite/accepted: 2015.12 .21

\section{R E S U M O}

O objetivo deste trabalho foi selecionar genótipos de capim-elefante com base na produção de matéria seca, utilizando a metodologia de modelos mistos (REML/BLUP). O experimento foi conduzido na Pesagro, RJ, Brasil. Foram obtidos 25 híbridos em esquema de dialelo parcial, 10 genitores e a cultivar Capim Cana D'África. Foram avaliadas as características largura da folha (LL), comprimento da folha (CF), diâmetro do colmo (DC) e número de perfilhos (NP) em seis semanas a partir da $5^{\text {a }}$ semana após o plantio. Na última semana foram avaliadas a porcentagem (\%MS) e produção de matéria seca (PMS). Os componentes de variância e coeficientes de repetibilidade foram estimados pelo método da máxima verossimilhança restrita (REML) e a predição de valores genotípicos pela melhor predição linear não viciada (BLUP). Para análise das variáveis foi utilizado o software Selegen-REML/BLUP. Observou-se grande influência da variância ambiental na variância fenotípica para as características LL, CF, DC e NP. Foram obtidos valores de repetibilidade de média magnitude para a LL, CF e DC. Os genótipos que apresentaram potencial para maior produção de biomassa foram H16, H17, G, P1, H4, P4, H25 e H22.

Palavras-chave: características morfoagronômicas, melhoramento de plantas, modelos mistos, Pennisetum purpureum.

\begin{abstract}
A B S T R A C T
The objective of this work was to select elephant grass genotypes based on dry matter production, using the mixed model methodology (REML/BLUP). The experiment was conducted at Pesagro, RJ, Brazil. Twenty five hybrids were obtained in partial diallel scheme, 10 parents and the cultivar Capim Cana D'Africa. We evaluated the characteristics sheet width (LL), leaf length (CF), stem diameter (DC) and number of tillers (NP) in six weeks from the fifth week after planting. The percentage (\% MS) and dry matter production (PMS) was evaluated in the last week. The components of variance and repeatability coefficients were estimated by restricted maximum likelihood (REML) and the prediction of phenotypic and genotypic values for the best linear unbiased prediction (BLUP). The Selegen-REML/BLUP software was used for analysis of the variables. There was great influence of environmental variance in phenotypic variance for traits, CF, DC and NP. Average magnitude of repeatability values were obtained for the LL, CF and DC. The genotypes that presented potential for greater production of biomass were H16, H17, G, P1, H4, P4, H25 and H22.
\end{abstract}

Key-words: mixed models, morpho-agronomic traits, Pennisetum purpureum, plant breeding. 


\section{INTRODUÇÃO}

O capim-elefante (Pennisetum purpureum Schum.) é uma forrageira perene, com alta produção de matéria seca e utilizada na alimentação de animais em rebanho leiteiro e de corte (Lima et al., 2010). Devido a essa alta produção de matéria seca tem sido utilizada também para fins energéticos, sendo que os genótipos devem apresentar maior teor de fibras, ao contrário dos genótipos destinados a alimentação animal que devem apresentar baixos teores de fibras e altos teores de proteína (Oliveira et al., 2012).

No Brasil, segundo a Empresa de Pesquisa Energética (2014) a biomassa é responsável por 7,6\% do suprimento de energia elétrica do país, ou seja, a terceira maior fonte de energia. $\mathrm{O}$ uso da biomassa de capim-elefante na matriz energética nacional pode permitir a geração de eletricidade e a produção de biocombustíveis onde a construção de hidrelétricas e o cultivo de biomassas tradicionais não são possíveis (Chies, 2015).

Existe a necessidade de genótipos de capim-elefante adaptadas aos diferentes ecossistemas (Souza Sobrinho et al., 2005). O melhoramento do capim-elefante com base no vigor híbrido permite fixar determinado genótipo e depois multiplicá-lo via propagação vegetativa (Pereira et al., 2006). Pesquisas cujo objetivo é a busca por genótipos com características desejáveis para futuros programas de melhoramento de capim-elefante para fins energéticos já são realizadas pela Empresa Brasileira de Pesquisas Agropecuárias (Chies, 2015).

A estimação de componentes da variância por máxima verossimilhança restrita (REML) e a predição de valores genéticos pela melhor predição linear não viciada (BLUP) são os procedimentos analíticos recomendados em genética quantitativa e seleção em plantas perenes (Resende et al., 2001). A utilização de modelos mistos fornece estimativas mais precisas da variação genética e dos valores genéticos individuas, pois permite a predição acurada e não enviesada dos valores genéticos mesmo sob desbalanceamento. Isso também facilita o uso simultâneo das informações do indivíduo, da família e de medidas repetidas no tempo (Pereira et al., 2013).

Diante do exposto, o objetivo deste trabalho foi selecionar genótipos de capim-elefante com base na produção de matéria seca, utilizando a metodologia de modelos mistos (REML/BLUP).

\section{MATERIAL E MÉTODOS}

O experimento foi realizado na estação experimental do Centro Estadual de Pesquisa em Agroenergia e Aproveitamento de Resíduos, Pesagro-RJ, em Campos dos Goytacazes, na região Norte Fluminense do Estado do Rio de Janeiro, Brasil, situada a $21^{\circ} 19^{\prime} 23^{\prime \prime}$ de latitude Sul e $41^{\circ} 19^{\prime} 40^{\prime \prime}$ de longitude Oeste, com altitude de $20 \mathrm{~m}$, e o clima classificado como do tipo Aw de Koppen. O solo é classificado como argissolo distrófico (Embrapa, 2006). O solo apresenta as seguintes características: $\mathrm{pH} \mathrm{CaCl}=$ 6,0; fósforo $=11 \mathrm{mg} \mathrm{dm}^{-3}$; potássio $=3,2 \mathrm{mmol}_{\mathrm{c}} \mathrm{dm}^{-3}$; Cálcio $=46,8 \mathrm{mmol}_{\mathrm{c}} \mathrm{dm}^{-3} ;$ Magnésio $=22,5 \mathrm{mmol}_{\mathrm{c}}$ $\mathrm{dm}^{-3}$; Alumínio $=0,0 \mathrm{cmol}_{\mathrm{c}} \mathrm{dm}^{-3} ;$ Hidrogênio + Alumínio $=21,2 \mathrm{mmol}_{\mathrm{c}} \mathrm{dm}^{-3} \mathrm{e}$ Carbono $=12,3 \mathrm{~g} \mathrm{dm}^{-3}$.

Foram avaliados 25 híbridos de capim-elefante obtidos em esquema de dialelo parcial (Quadro 1) e mais onze genótipos, os 10 genitores e a cultivar Capim Cana D'África (G). Os genitores e a cultivar Capim

Quadro 1 - Esquema de cruzamentos do dialelo parcial com dez genitores Esquema de cruzamentos do dialelo parcial com dez genitores

\begin{tabular}{|c|c|c|c|c|c|}
\hline \multirow[b]{2}{*}{$\begin{array}{l}\text { Genitores } \\
\text { masculinos }\end{array}$} & \multicolumn{5}{|c|}{ Genitores femininos } \\
\hline & $\begin{array}{l}\text { Cubano } \\
\text { Pinda } \\
\text { (P1) }\end{array}$ & $\begin{array}{c}\text { Cameroon - } \\
\text { Piracicaba } \\
\text { (P2) }\end{array}$ & $\begin{array}{c}\text { P241- } \\
\text { Piracicaba } \\
\text { (P3) }\end{array}$ & $\begin{array}{c}\text { IAC- } \\
\text { Campinas } \\
\text { (P4) }\end{array}$ & $\begin{array}{c}\text { Guaçu/ } \\
\text { IZ.2 } \\
\text { (P5) }\end{array}$ \\
\hline Mercker (P6) & $\mathrm{H} 1$ & H6 & H11 & H16 & $\mathrm{H} 21$ \\
\hline Três Rios (P7) & $\mathrm{H} 2$ & H7 & H12 & H17 & $\mathrm{H} 22$ \\
\hline $\begin{array}{l}\text { Mercker 86- } \\
\text { México (P8) }\end{array}$ & $\mathrm{H} 3$ & $\mathrm{H} 8$ & H13 & $\mathrm{H} 18$ & $\mathrm{H} 23$ \\
\hline $\begin{array}{c}\text { Taiwan } \\
\text { A-144 (P9) }\end{array}$ & $\mathrm{H} 4$ & H9 & $\mathrm{H} 14$ & H19 & $\mathrm{H} 24$ \\
\hline Roxo (P10) & H5 & H10 & H15 & $\mathrm{H} 20$ & H25 \\
\hline
\end{tabular}


Cana D'África foram selecionados com base na divergência genética (Lima et al., 2011) e nas características morfoagronômicas, preferencialmente aqueles com maior produção de biomassa (Rossi, 2010).

O delineamento experimental utilizado foi em blocos ao acaso, com três repetições. A unidade experimental foi constituída por uma linha de $6 \mathrm{~m}$ de comprimento e $1,5 \mathrm{~m}$ entre linhas, totalizando $9 \mathrm{~m}^{2}$. A área útil foi de $7,5 \mathrm{~m}^{2}$, excluindo $0,75 \mathrm{~m}^{2} \mathrm{de}$ cada uma das extremidades. O plantio foi realizado em junho de 2012, utilizado colmos inteiros de $1 \mathrm{~m}$ (três gemas por colmo), distribuídos nos sulcos no sistema "pé com ponta". Foi realizada a adubação no plantio com $100 \mathrm{~kg}^{-1}$ de $\mathrm{P}_{2} \mathrm{O}_{5}$ (Superfosfato Simples).

Foram feita avaliações na $5^{\underline{a}}, 6^{\underline{a}}, 7^{\mathrm{a}}, 8^{\mathrm{a}}, 10^{\mathrm{a}} \mathrm{e}$ e $12^{\mathrm{a}}$ semana após o plantio. As características avaliadas foram aquelas correlacionadas com a produção de matéria seca (Menezes et al., 2014): largura da folha (LL, cm) utilizando régua graduada tomando ao acaso cinco plantas de cada parcela; comprimento da folha (CF, $\mathrm{cm}$ ) - tomando ao acaso cinco folhas inteiras de cinco plantas de cada parcela, foi mensurada com o auxílio de régua graduada na primeira folha inteira; diâmetro do colmo (DC, $\mathrm{mm}$ ) - mensurado com o auxílio de um paquímetro, tomando ao acaso cinco perfilhos de cinco plantas de cada parcela; número de perfilhos (NP) - foi realizada a contagem de todos os perfilhos da parcela.

Na última semana de avaliação todos os perfilhos da área útil de cada parcela foram pesados e, coletadas amostras de três perfilhos. Essas amostras foram passadas na picadeira e mantidas em estufa a $65^{\circ} \mathrm{C} / 72 \mathrm{~h}$ para a determinação da amostra seca ao ar (ASA). Posteriormente, esse material foi moído em peneira de $1 \mathrm{~mm}$ e mantidos em estufa a $105^{\circ} \mathrm{C} / 12 \mathrm{~h}$ para a determinação da amostra seca em estufa (ASE). Com os valores de ASA e ASE foram obtidos a porcentagem de matéria seca (\%MS). Com os valores do peso de perfilhos e \%MS foi possível obter a produção de matéria seca (PMS), expressa em $t$ ha $^{-1}$.

Os componentes de variância e coeficientes de repetibilidade foram estimados pelo método da máxima verossimilhança restrita (REML) e a predição de valores genotípicos pela melhor predição linear não viciada (BLUP). Para análise das variáveis foi utilizado o software SelegenREML/BLUP (Resende, 2007).
Foi utilizado o modelo 28 para análise das características avaliadas nas seis semanas (LL, CF, DC e NP). Esse modelo compreende medidas repetidas com delineamento em blocos ao acaso, onde se utiliza a média da parcela para cada característica. De acordo com Resende (2007), o modelo estatístico é expresso por: $y=X m+Z g+$ $W p+e$, em que $y$ é o vetor de dados, m é o vetor dos efeitos das combinações medição-repetição (assumidos como fixos) somados à média geral, $g$ é o vetor dos efeitos genotípicos (assumidos como aleatórios), $p$ é vetor dos efeitos de ambiente permanente (parcelas) (aleatórios) e $e$ é o vetor de erros ou resíduos (aleatórios). As letras maiúsculas representam as matrizes de incidência para os referidos efeitos.

O modelo 96 foi utilizado para analisar as características avaliadas apenas na ultima semana (\%MS e PMS). Esse modelo corresponde o delineamento em blocos ao acaso, com uma planta por parcela e desprezando o parentesco entre os genótipos avaliados. $\mathrm{O}$ modelo estatístico nesse caso é expresso por: $y=X r+Z g+e$, em que $y$ é o vetor de dados, $r$ é o vetor que contém a média geral e os efeitos de repetição, $g$ é o vetor dos efeitos genotípicos (assumidos como aleatórios), $e$ é o vetor de erros ou resíduos (aleatórios). As letras maiúsculas representam as matrizes de incidência para os referidos efeitos (Resende, 2007).

A heritabilidade de parcelas individuais no sentido amplo foi estimada por: $h_{g}{ }^{2}=\mathrm{Vg} / \mathrm{Vf}$

A repetibilidade ao nível de parcelas foi calculada por:

$r=\left(V_{g}+V_{\text {perm }}\right) / V_{f}$

Em que

$V_{g}$ - variância genotípica;

$V_{\text {perm }}$ - variância de ambiente permanente entre parcelas;

$V_{f}$ - variância fenotípica individual. 


\section{RESULTADOS E DISCUSSÃO}

Nas quatro características avaliadas observouse grande influencia da variância ambiental na variância fenotípica. Isso significa que as características foram bastante influenciadas por fatores ambientais. Esse resultado pode ser corroborado pelos baixos valores de heritabilidade $\left(h_{g}{ }^{2}\right)$. Em relação à repetibilidade $(r)$ verificou-se para as características LL, CF, DC e NP valores de 0,2239; 0,5574; 0,4454 e 0,5294, respectivamente (Quadro 2). Segundo Resende (2002) repetibilidade entre 0,30 e 0,60 são consideradas medianas. Com isso, observa-se que apenas a característica LL apresentou baixa repetibilidade.

Em relação às características porcentagem (\%MS) e a produção de matéria seca (PMS) observou-se grande participação da variância genotípica nas diferenças entre fenótipos (Quadro 3). Os valores de $h_{g}{ }^{2}$ apresentados pelas duas características foram de 0,6924 e 0,7306 com desvios de 0,2265 e 0,2326 , respectivamente. Valores de $h_{g}{ }^{2}$ acima de 0,46 evidenciam grande potencial de seleção (Garcia e Nogueira, 2005). De acordo com os valores de coeficiente de variação genotípica ( $\mathrm{CV}_{g i}$ ), principalmente para PMS, pode-se afirmar que a idade de corte foi suficiente para a manifestação das diferenças entre os genótipos avaliados.

No Quadro 4 encontram-se os valores genotípicos e ganhos genéticos com a seleção dos oito melhores genótipos de capim-elefante para as características \%MS e PMS. Verifica-se que os híbridos H16 (IACCampinas x Mercker), H17 (IAC-Campinas x Três
Rios) e H25 (Guaçu/IZ.2 x Roxo) estiveram entre os primeiros oito melhores classificados para as duas características. O mesmo ocorreu para o genitor $\mathrm{P} 4$ (IAC-Campinas). Observa-se que o genótipo P4 foi o genitor feminino dos híbridos H16 e H17. Este se apresentou entre os oito melhores genótipos, mas não em ordem superior aos híbridos H16 e H17. Os genótipos P6 (Mercker) e P7 (Três Rios) que foram os genitores dos híbridos $\mathrm{H} 16$ e H17, respectivamente, não estiveram entre os oito melhores genótipos para as características \%MS e PMS. De acordo com Menezes et al. (2015) o genótipo Mercker apresenta destacados valores de efeito de variedade para a característica PMS e pode ser utilizado para compor a população base para futuros cruzamentos promissores em capimelefante. Em Lima et al. (2011) os genótipos P4 (IACCampinas), P6 (Mercker) e P7 (Três Rios) foram classificados em diferentes grupos heteróticos. Enquanto os genótipos Mercker e Três Rios estiveram no grupo I, o IAC-Campinas esteve no grupo III, ou seja, distantes geneticamente. Desta forma pode-se dizer que a divergência genética existente ente os genótipos IAC-Campinas $x$ Mercker e IAC-Campinas $x$ Três Rios contribuiu para a heterose nesses cruzamentos.

Em relação apenas a PMS que os genótipos que se destacaram foram: $\mathrm{H} 4$ (Cubano Pinda $\mathrm{x}$ Taiwan A-144), H16 (IAC-Campinas x Mercker), H17 (IACCampinas x Três Rios), P1 (Cubano Pinda), P4 (IACCampinas), H22 (Guaçu/IZ.2 x Três Rios), H25 (Guaçu/IZ.2 x Roxo) e G (Capim Cana D'África). No caso dos híbridos $\mathrm{H} 22$ e H25 foi observada a presença de heterose para a característica PMS,

Quadro 2 - ativas de parâmetros genéticos para largura da folha (LL, cm), comprimento da folha (CF, cm), diâmetro do colmo (DC, mm) e número de perfilhos (NP) em 36 genótipos de capim-elefante, no município de Campos dos Goytacazes, RJ, Brasil

\begin{tabular}{ccccc}
\hline Parâmetro & LL & CF & DC & NP \\
\hline$V_{g}$ & 0,0141 & 7,2681 & 0,2666 & 1,6791 \\
$V_{\text {perm }}$ & 0,0184 & 61,4176 & 1,3884 & 12,6214 \\
$V_{e}$ & 0,1128 & 54,5192 & 2,0604 & 12,7117 \\
$V_{f}$ & 0,1454 & 123,2049 & 3,7155 & 27,0123 \\
$h_{g}^{2}$ & 0,0972 & 0,0589 & 0,0717 & 0,0621 \\
& $+/-0,0347^{1}$ & $+/-0,0270^{1}$ & $+/-0,0298^{1}$ & $+/-0,0277$ \\
$r$ & 0,2239 & 0,5574 & 0,4454 & 0,5294 \\
& $+/-0,0526^{1}$ & $+/-0,0830^{1}$ & $+/-0,0742^{1}$ & $+/-0,0808$ \\
$c_{\text {perm }}^{2}$ & 0,1267 & 0,4985 & 0,3736 & 0,4672 \\
Média geral & 2,4840 & 59,0158 & 11,8239 & 13,7263 \\
\hline
\end{tabular}

${ }_{1}^{1}$ Desvio-padrão das estimativas; $\mathrm{V}_{\mathrm{g}}$ - variância genotípica; $\mathrm{V}_{\text {perm }}$ - variância de ambiente permanente entre parcelas; $V_{e}$ - variância residual; $V_{f}$ - variância fenotípica individual; $h^{2}$ - heritabilidade de parcelas individuais no sentido amplo; $r$ - repetibilidade ao nível de parcelas; $c^{2}{ }_{\text {perm }}$ - coeficiente de determinação dos efeitos de ambiente permanente. 
Quadro 3 - Estimativas de parâmetros genéticos para porcentagem de matéria seca (\%MS) e produção de matéria seca (PMS, t ha-1) em 36 genótipos de capim-elefante, no município de Campos dos Goytacazes, RJ, Brasil

\begin{tabular}{ccc}
\hline Parâmetro & \%MS & PMS \\
\hline$V_{g}$ & 13,21971 & 1,8794 \\
$V_{\theta}$ & 5,8723 & 0,6929 \\
$V_{f}$ & 19,0920 & 2,5724 \\
$h_{g}^{2}$ & $0,6924+/-0,2265^{1}$ & $0,7306+/-0,2326^{1}$ \\
$C V_{g i}(\%)$ & 16,8529 & 31,6670 \\
$C V_{\theta}(\%)$ & 11,2323 & 19,2291 \\
$C V_{r}(\%)$ & 1,5003 & 1,6468 \\
Média geral & 21,5741 & 4,3291 \\
\hline
\end{tabular}

${ }^{1}$ Desvio-padrão das estimativas; $V_{g}$ - variância genotípica; $V_{e}$ - variância residual; $V_{f}$ - variância fenotípica individual; $h^{2}$ - heritabilidade de parcelas individuais no sentido amplo; $C V_{g i}(\%)$ - coeficiente de variação genotípica; $C V_{e}(\%)$ - coeficiente de variação residual; $C V_{r}(\%)$ - coeficiente de variação relativa.

Quadro 4 - Valores genotípicos e ganhos genéticos com a seleção dos 8 melhores genótipos de capim-elefante, para as características porcentagem (\%MS) e produção de matéria seca (PMS, $t^{\text {ha-1)}}{ }^{1}$, no município de Campos dos Goytacazes, RJ, Brasil

\begin{tabular}{|c|c|c|c|c|c|c|c|c|c|c|}
\hline \multirow{2}{*}{$\begin{array}{l}\text { Or- } \\
\text { dem }\end{array}$} & \multicolumn{5}{|c|}{$\%$ MS } & \multicolumn{5}{|c|}{ PMS } \\
\hline & Gen. & g & $\mathbf{u}+\mathbf{g}$ & ganho & $\begin{array}{l}\text { Nova } \\
\text { média }\end{array}$ & Gen. & g & $\mathbf{u}+\mathbf{g}$ & ganho & $\begin{array}{l}\text { Nova } \\
\text { média }\end{array}$ \\
\hline 1 & G8 & 7,00 & 28,57 & 7,00 & 28,57 & H16 & 3,80 & 8,13 & 3,80 & 8,13 \\
\hline 2 & H17 & 6,67 & 28,25 & 6,83 & 28,41 & H17 & 2,53 & 6,86 & 3,16 & 7,49 \\
\hline 3 & H16 & 5,31 & 26,88 & 6,33 & 27,90 & G11 & 2,16 & 6,49 & 2,83 & 7,16 \\
\hline 4 & $\mathrm{H} 21$ & 4,90 & 26,48 & 5,97 & 27,54 & G1 & 2,12 & 6,44 & 2,65 & 6,98 \\
\hline 5 & $\mathrm{H} 25$ & 4,86 & 26,43 & 5,75 & 27,32 & H4 & 1,64 & 5,97 & 2,45 & 6,78 \\
\hline 6 & H13 & 3,48 & 25,05 & 5,37 & 26,94 & G4 & 1,38 & 5,71 & 2,27 & 6,60 \\
\hline 7 & G4 & 3,12 & 24,70 & 5,05 & 26,62 & $\mathrm{H} 25$ & 1,00 & 5,33 & 2,09 & 6,42 \\
\hline 8 & H8 & 2,95 & 24,52 & 4,79 & 26,36 & H22 & 0,97 & 5,30 & 1,95 & 6,28 \\
\hline
\end{tabular}

Em que: Gen - genótipo; $\mathrm{g}$ - efeito genotípico predito; u + g: média genotípica ou valores genotípicos.

pois seus genitores não se encontram entre os oito melhores selecionados. Ao contrário do que ocorreu no presente estudo, em Oliveira et al. (2014), avaliando a produção anual de genótipos de capim-elefante, o genótipo Guaçu/IZ.2, genitor feminino dos híbridos H22 e H25, esteve entre os genótipos com maior PMS (grupo elite).

\section{CONCLUSÕES}

Pode-se concluir que há grande influência da variância ambiental na variância fenotípica para as características LL, CF, DC e NP, ao contrário do que ocorreu para as características \%MS e PMS, onde foi verificada grande influência da variância genotípica na variância fenotípica. Com base nos valores genotípicos e ganhos genéticos para a PMS do presente estudo, pode-se selecionar os genótipos H4 (Cubano Pinda x Taiwan A-144), H16 (IACCampinas $x$ Mercker), H17 (IAC-Campinas $x$ Três Rios), P1 (Cubano Pinda), P4 (IAC-Campinas), H22 (Guaçu/IZ.2 x Três Rios), H25 (Guaçu/IZ.2 x Roxo) e G (Capim Cana D’África) quando o objetivo for a maior produção de biomassa em capim-elefante.

\section{AGRADECIMENTO}

A Fundação de Amparo a Pesquisa do Estado do Rio de Janeiro (FAPERJ) pelo auxílio financeiro. 


\section{REFERÊNCIAS BIBLIOGRÁFICAS}

Chies, V. (2015) - Pesquisa investe em capim como fonte de energia. Empresa Brasileira de Pesquisa Agropecuária - Embrapa. Disponível em: <https://www.embrapa.br/busca-de-noticias/-/noticia/2422024/pesquisainveste-em-capim-como-fonte-de-energia>.

Embrapa - Empresa Brasileira de Pesquisa Agropecuária. (2006) - Sistema brasileiro de classificação de solos, Rio de Janeiro, EMBRAPA. 412 p.

Empresa de Pesquisa Energética (Brasil). (2014) - Balanço Energético Nacional 2014: Ano base 2013. Rio de Janeiro: EPE. 288p.

Garcia, C.H. e Nogueira, M.C.S. (2005) - Utilização da metodologia REML/BLUP na seleção de clones de eucalipto. Scientia Forestalis, n. 68, p. 107-112.

Lima, E.S.; Silva, J.F.C.; Vásquez, H.M.; Andrade, E.N.; Dominicis, B.B.; Morais, J.P.G.; Costa, D.P.B. e Araújo, S.A.C. (2010) - Características agronômicas e nutritivas das principais cultivares de capim-elefante do Brasil. Veterinária e Zootecnia, vol. 17, n. 3, p. 324-334.

Lima, R.S.N.; Daher, R.F.; Gonçalves, L.S.A.; Rossi, D.A.; Amaral Júnior, A.T.; Pereira, M.G. e Lédo, F.J.S. (2011) - RAPD and ISSR markers in the evaluation of genetic divergence among accessions of elephant grass. Genetics and Molecular Research, vol. 10, n. 3, p. 1304-1313. http://dx.doi.org/10.4238/vol10-3gmr110

Menezes, B.R.S.; Daher, R.F.; Gravina, G.A.; Amaral Júnior, A.T.; Oliveira, A.V.; Schneider, L.S.A. e Silva, V.B. (2014) - Correlações e análise de trilha em capim-elefante para fins energéticos. Revista Brasileira de Ciências Agrárias, vol. 9, n. 3, p. 465-470. http://dx.doi.org/10.5039/agraria.v9i3a3877

Menezes, B.R.S.;Daher,R.F.;Gravina, G.A.;Pereira,A.V.;Sousa, L.B.;Rodrigues, E.V.;Silva, V.B.;Gottardo,R.D.; Schneider, L.S.A. e Novo, A.A.C. (2015) - Estimates of heterosis parameters in elephant grass (Pennisetum purpureumSchumach.)forbioenergy production.ChileanJournalofAgricultural Research, vol.75,n.4, p.395-401. http://dx.doi.org/10.4067/S0718-58392015000500003

Oliveira, E.S.; Daher, R.F; Tunes, E.N.; Soares, R.T.R.N.; Gonçalves, A.C.S. e Gravina, G.A. (2012) - Potencial de germinação de estacas e avaliação de características morfoagronômicas em seis cultivares de capimelefante (Pennisetum purpureum Schum.) para fins energéticos em Campos dos Goytacazes, RJ. Natureza on line, vol. 10, n. 1, p. 39-45.

Oliveira, M.L.F.;Daher,R.F.;Gravina, G.A.;Silva, V.B.;Viana, A.P.;Rodrigues, E.V.;Shimoya, A.;Amaral Júnior, A.T.; Menezes, B.R.S. e Rocha, A.S. (2014) - Pre-breeding of elephant grass for energy purposes and biomass analysisinCamposdosGoytacazes-RJ,Brazil. AfricanJournalofAgricultural Research, vol.9,n.36, p. 2743-2758. http://dx.doi.org/10.5897/AJAR2014.8900

Pereira, A.V.; Daher, R.F.; Pereira, M.G.; Lédo, F.J.S.; Sobrinho, F.S.; Amaral Junior, A.T.; Freitas, V.P.; Pereira, T.N.S. e Ferreira, C.F. (2006) - Análise de cruzamentos dialélicos entre capim- Análise de cruzamentos dialélicos entre capim-elefante (Pennisetum purpureum Schum.) e milheto (Pennisetum glaucum (L.) R. Br.). 1. Características morfoagronômicas. Acta Scientiarum - Agronomy, vol. 28, n. 2, p. 267-275. http://dx.doi.org/10.4025/actasciagron.v28i2.1128

Pereira, T.B.; Carvalho, J.P.F.; Botelho, C.E.; Resende, M.D.V.; Rezende, J.C. e Mendes, A.N.G. (2013) Eficiência da seleção de progênies de café $\mathrm{F}_{4}$ pela metodologia de modelos mistos (REML/BLUP). Bragantia, vol. 72, n. 3, p. 230-236. http://dx.doi.org/10.1590/brag.2013.031

Resende, M.D.V.; Furlani-Junior, E.; Moraes, M.L.T. e Fazuoli, L.C. (2001) - Estimativas de parâmetros genéticos e predição de valores genotípicos no melhoramento do cafeeiro pelo procedimento REML/ BLUP. Bragantia, vol. 60, n. 3, p. 185-193. http://dx.doi.org/10.1590/S0006-87052001000300005

Resende, M.D.V. (2002) - Genética biométrica e estatística no melhoramento de plantas perenes. Brasília, Embrapa Informação Tecnológica. 975 p.

Resende, M.D.V. (2007) - Software SELEGEN REML/BLUP: sistema estatístico e seleção genética computadorizada via modelos lineares mistos. Colombo, Embrapa Florestas. 359 p.

Rossi, D.A. (2010) - Avaliação morfoagronômica e da qualidade da biomassa de acessos de capim-elefante (Pennisetum purpureum Schum.) para fins energéticos no norte fluminense. Dissertação de Mestrado. Universidade Estadual do Norte Fluminense, Brasil. 66 p.

Souza Sobrinho, F.; Pereira, A.V.; Ledo, F.J.S.; Botrel, M.A.; Oliveira, J.S. e Xavier, D.F. (2005) - Avaliação agronômica de híbridos interespecíficos entre capim-elefante e milheto. Pesquisa Agropecuária Brasileira, vol. 40, n. 9, p. 873-880. http://dx.doi.org/10.1590/S0100-204X2005000900006 